\section{Lateral Inhibition}

Ronald Cohen

Center for Cognitive Aging and Memory, University of Florida, Gainsville, FL, USA

\section{Synonyms}

Sensory inhibition; Spatial inhibition

\section{Definition}

Lateral inhibition refers to the capacity of excited neurons to reduce the activity of their neighbors. Neurons that are firing inhibit the stimulation of surrounding. Accordingly, only the neurons that are most stimulated and least inhibited respond. Lateral inhibition plays an important role in visual perception by increasing the contrast and resolution of visual stimuli. This occurs at various levels of the visual system. For example, when a small light is presented in a dark environment, receptors on the retina central to the stimulus are activated and transduce the visual information to the brain, while receptors that are peripheral to the stimulus send inhibitory signals that enhance the perception of darkness in the surrounding. This process has the effect of creating greater dark-light contrast and is responsible for the Mach band visual effect. Similar inhibitory processes occur cortically and contribute to both object and spatial perception.

\section{Cross-References}

- Cortical Magnification

$\checkmark$ Enhancement

- Visual Perception

\section{References and Readings}

Ratliff, F., Knight, B. W., Toyoda, J., \& Hartline, H. K. (1967). Enhancement of flicker by lateral inhibition. Science, 158(3799), 392.

Von Békésy, G. (1967). Sensory inhibition. Princeton: Princeton University Press. 